\title{
Mercury versus Oscillometric Device in Measuring Blood Pressure in Elderly: Which Is Authentic?
}

\author{
Vathulan Sujanitha*, Thirunavukarasu Kumanan, Mahesan Guruparan, \\ Thavakularatnam Inthuja \\ Teaching Hospital-Jaffna, Jaffna, Srilanka \\ Email: *sujanitha@yahoo.com, mtkumanan@yahoo.com, mahesanguruparan@yahoo.com, \\ inthuja22@gmail.com
}

How to cite this paper: Sujanitha, V., Kumanan, T., Guruparan, M. and Inthuja, T. (2018) Mercury versus Oscillometric Device in Measuring Blood Pressure in Elderly: Which Is Authentic? Open Access Library Journal, 5: e4353.

https://doi.org/10.4236/oalib.1104353

Received: January 19, 2018

Accepted: February 8, 2018

Published: February 11, 2018

Copyright () 2018 by authors and Open Access Library Inc.

This work is licensed under the Creative Commons Attribution International License (CC BY 4.0).

http://creativecommons.org/licenses/by/4.0/

\begin{abstract}
Aim: To compare the systolic and diastolic blood pressure in both arms in elderly patients by mercury sphygmomanometer and oscillometric recorders adopting standard practice of measuring blood pressure. Materials and methods: It is a cross sectional study done in 204 elderly patients aged more than 60 years over a period of two months at teaching hospital, Jaffna, Sri Lanka. Blood pressure was measured in both arms by both devices adopting the standard practice of blood pressure measurements. Results: The mean Systolic Blood Pressure (SBP) by oscillometric recorder on both right and left arm was $4.18 \mathrm{mmHg}$ and $3.66 \mathrm{mmHg}$ higher than mercury sphygmomanometer readings. The mean Diastolic Blood Pressure (DBP) by oscillometric recorder on both right arm and left arm was 1.67 and $1.64 \mathrm{mmHg}$ lower than mercury sphygmomanometer readings. There wasn't any significant difference in inter arm blood pressure measurement. The study group consisted of $54.3 \%$ males and $45.7 \%$ of females. The mean age of the study group was 69.46 years. Conclusion: Though there is no much difference observed in the gross values in reading the blood pressure by both oscillometric recorders and mercury sphygmomanometers, there is statistically significant discrepancy in blood pressure readings particularly in the elderly population which needs to be studied further in detail.
\end{abstract}

\section{Subject Areas}

Cardiology, Geriatrics, Public Health

\section{Keywords}

Oscillometric, Blood Pressure, Elderly Mercury, Sphygmomanometer 


\section{Introduction}

Hypertension is an important risk factor for most of the common causes of death in Sri Lanka; coronary heart disease, stroke, heart and renal failure. Approximately one-fifth to one-third of adult Sri Lankan population has blood pressure levels that are above normal and the prevalence is similar to those in most of the developed countries [1] [2] and [3]. Blood pressure levels are strongly associated with the morbidity and mortality of cardiovascular diseases. Hence, measuring blood pressure and treating is of utmost importance in managing a patient's cardiovascular risk [4]. Even though ambulatory blood pressure readings are superior to office and home blood pressure readings [5] the latter one is preferred as it is simple, freely available and cost effective. Conventional mercury sphygmomanometers and oscillometric electronic blood pressure recorders are widely used for this purpose [6]. Mercury sphygmomanometers have been considered as a gold standard for measuring blood pressure reading for a long time. However in the recent past oscillometric equipments had gain importance [7]. Considering the health hazards and the environmental safety, the Sri Lankan ministry of health made a national policy to sanction the import of mercury equipments including sphygmomanometers into the country. From a clinician's point of view, there are unexplored pros and cons in switching to oscillometric sphygmomanometers from mercury ones [8] [9]. As both the manometers are made up of two totally different physical principles in measuring blood pressure, it is anticipated that the blood pressure values could differ particularly in elderly patients due to their vascular stiffness, which is believed to be the cause for the isolated systolic hypertension of elders. This study is designed to look in to the differences of systolic and diastolic blood pressures in elderly patients by both methods adopting standard practice of measuring blood pressure.

\section{Methods}

This is a cross sectional study, included 204 patients admitted over a period of two months at Teaching hospital, Jaffna, Sri Lanka. Patients aged more than 60 years admitted to medical units during the period of October and November, 2016 were included. Bed bound patients (bed bound and was not able to walk even with support) and critically ill patients (those who need organ support including inotropes, ventilation or renal replacement therapy) were excluded from this study.

Ethical approval was sought and obtained from the Ethical Review Committee, Faculty of Medicine, and University of Jaffna, Sri Lanka.

\section{Assessment of blood pressure}

Blood pressure of the patients was measured by both mercury sphygmomanometer and oscillometric (validated Omron EN-M3) electronic blood pressure recorder. After about 10 minutes of quiet sitting, the blood pressure was measured in sitting position and the arm was placed on the table. The mercury sphygmo- 
manometer cuff (appropriate size) was wrapped around right arm first and then left arm followed by oscillometric recorder. Three readings were taken in each arm with oscillometric recorders and the average was taken for the analysis [10].

\section{Data Analysis}

The data was analyzed using SPSS (version 24) statistical package. Descriptive statistics like frequency, percentage, mean and standard deviation were used to summarize the data and t-test was used to assess the significance between right and left arm blood pressure by using both mercury and oscillometric blood pressure recorders.

\section{Results}

A total of 204 patients aged more than 60 years were analyzed out of which $54.3 \%$ (110 patients) were males and 45.7\% (94 patients) were females.64.5\% (131 patients) of the study group were known to have hypertension and $35.5 \%$ (73 patients) were not known to have hypertension. The mean age of the study group was 69.46 years. The mean SBP by mercury sphygmomanometer on both right and left arm were $128.25 \mathrm{mmHg}$ and $127.83 \mathrm{mmHg}$ respectively without any significant inter arm difference. The mean DBP by mercury sphygmomanometer on both right arm and left arm were 74.45 and $74.09 \mathrm{mmHg}$ respectively.

Similarly the mean SBP by oscillometric sphygmomanometer on both right and left arm were 132.44 and $131.49 \mathrm{mmHg}$ which were significantly higher than the mercury readings $(\mathrm{p}<0.01)$. The mean DBP by oscillometric sphygmomanometer on both right and left arm were 72.78 and $72.45 \mathrm{mmHg}$ which were lower than the mercury readings (Table 1, Table 2). High blood pressure, both systolic and diastolic was noted by oscillometric recorders when the heart rate was high (Table 3 ).

\section{Discussion}

Though the gold standard measurement of arterial blood pressure is direct intra-

Table 1. Mean blood pressure readings of right and left arm by both methods (paired samples descriptive statistics).

\begin{tabular}{ccccc}
\hline Pair & Variable & N & Mean & Standard Deviation \\
\hline Pair 1 & MSR & 204 & 128.25 & 23.64 \\
& OSR & 204 & 132.44 & 22.77 \\
Pair 2 & MDR & 204 & 74.45 & 12.61 \\
& ODR & 204 & 72.78 & 12.08 \\
Pair 3 & MSL & 204 & 127.83 & 22.95 \\
& OSL & 204 & 131.49 & 22.72 \\
Pair 4 & MDL & 204 & 74.09 & 12.66 \\
& ODL & 204 & 72.45 & 12.19 \\
\hline
\end{tabular}


Table 2. Comparison of systolic and diastolic blood pressure by both devices (paired samples test).

\begin{tabular}{cccccc}
\hline Pair & Variable & Mean & $\begin{array}{c}\text { Standard } \\
\text { Deviation }\end{array}$ & $\begin{array}{c}\text { Standard Error } \\
\text { Mean }\end{array}$ & $\begin{array}{c}\text { Significant (2-tailed) } \\
\text { P-Value }\end{array}$ \\
\hline Pair 1 & MSR-OSR & -4.18 & 12.44 & 0.87 & 0.00 \\
Pair 2 & MDR-ODR & 1.67 & 8.86 & 0.62 & 0.01 \\
Pair 3 & MSL-OSL & -3.66 & 12.84 & 0.90 & 0.00 \\
Pair 4 & MDL-ODL & 1.64 & 8.88 & 0.62 & 0.01 \\
\hline
\end{tabular}

Table 3. Comparison of blood pressure measurement by oscillometric recorder with different pulse rate.

\begin{tabular}{cccccc}
\hline PR & N & OSR & ODR & OSL & ODL \\
\hline Pulse rate less than 72 & 44 & 131.89 & 69.61 & 129.05 & 69.30 \\
Pulse rate more than 72 & 160 & 132.59 & 73.65 & 132.16 & 73.31 \\
\hline
\end{tabular}

PR-Pulse Rate, N-Number, OSR-Oscillometric Systolic blood pressure of Right arm, ODR-Oscillometric Diastolic blood pressure of Right arm, OSL-Oscillometric Systolic Blood pressure of Left arm, ODL-Oscillometric Diastolic blood pressure of Left arm.

arterial measurement, it is not practical. We use indirect methods such as palpatory and auscultatory methods which are sufficiently accurate for many diagnostic and therapeutic studies [11]. Mercury sphygmomanometers are gradually being phased out, not because of technological advances but because of environmental concerns [12] [13]. It also has inter-observer differences which has a major impact on clinical assessment. Hence, oscillometric recorders are promoted among health care workers in Sri Lanka instead of mercury sphygmomanometers in recent times. The study was aimed to compare the differences in the measured blood pressure values with both these methods.

The mean systolic and diastolic blood pressure of the study group in both arms remains within the normal range irrespective of the method used. However, it is noted that the pulse pressure is increased in the study population when we used oscillometric recorders. Studies show that in the elderly the most powerful predictor of risk is increased pulse pressure due to decreased diastolic and increased systolic blood pressure [14]. Isolated systolic hypertension, an elevation of systolic but not diastolic blood pressure is the most prevalent type of hypertension in the elderly [15] and there is ample evidence to show that treating them will reduce the risk of cardiovascular events [14] [16]. Our study results also show a low normal diastolic and high normal systolic blood pressure compatible with previous studies.

The mean difference of the mercury and oscillometric readings in systolic blood pressure is $4.18 \mathrm{mmHg}$ in right arm and $3.66 \mathrm{mmHg}$ in left arm which are statistically significant. The reasons for high oscillometric readings are to be established, whether oscillometric records the initial subtle pressure waves conducted by the stiff vessels of the elderly needs further exploration. Many studies 
have compared mercury with oscillometric and digital devices and found oscillometric devices had better accuracy than the digital devices [17].

Similarly a small but statistically significant difference in measurement of diastolic blood pressure by both methods was found. Diastolic blood pressure by mercury sphygmomanometer was found to be higher than the oscillometric readings which may be attributed to low detection rates of korotkoff sounds by the observer. Phase 4 korotkoff sounds could have been picked up rather than the phase 5 (complete disappearance of sound) and the oscillometric recorder could have detected even the lowest intensity waves and given lower diastolic blood pressure.

There is not much inter-arm blood pressure difference by both methods. This is comparable to previous studies where minimum difference was observed [18]. The prevalence of inter-arm blood pressure difference could be low in the study population as it is very common in very elderly population with significant peripheral disease with multiple risk factors for atherosclerotic vascular disease to be present in both upper limbs [19].

It is observed that high blood pressure level was recorded when there is a high normal heart rate compared to subnormal heart rate. It's quite obvious that the recorder has more chance to pick up an early systolic and diastolic wave when the heart beats more often not giving a lag phase. When the heart rate is high, a high sympathetic drive is expected and obviously the blood pressure also tends to be high. Also physiologically, up to certain extent the elevation of heart rate increases cardiac output which accounts for a high blood pressure. These mechanisms would probably explain our findings. However, it needs further detailed study for better understanding.

\section{Conclusion}

Though there are not many differences observed in the gross values in reading the blood pressure by both oscillometric recorders and mercury sphygmomanometers, there are statistically significant discrepancies in reading the blood pressure in particular the elderly population. This needs expanded further research on different mercury sphygmomanometers and oscillometric devices to generalize the findings. This could have a great impact in the treatment of blood pressure in the post SPRINT era taking even subtle blood pressure levels into account in drawing guidelines and setting treatment goals.

\section{Limitations}

The limitations of this study are: 1) Number of subjects-For more reliable results the study can be done in expanded population; 2) Different brands of oscillometric devices could be employed in measuring the blood pressure and hence the results may vary in different studies; 3) The arterial pulse wave velocity also could be measured along with blood pressure measurements for better interpretation which was not done here. 


\section{Acknowledgements}

The authors thank the staff of medical wards and department of medicine of Teaching Hospital, Jaffna, Sri Lanka for the support given in data collection and analysis.

\section{Competing Interest}

The authors declare that there is no competing interest.

\section{Funding}

Self-funding for the materials is used.

\section{Consent for Publication}

Not applicable.

\section{Authors' Contribution}

TI and VS collected the data, TK and VS analyzed the data and are the main contributors in writing the report. All authors read and approved the final manuscript.

\section{Ethics, Consent and Permission}

The ethical approval for this study was obtained from Ethical review committee of Faculty of Medicine, University of Jaffna (Reference number J/ERC/16/73/NDR/0145) and informed written consent was taken from each participant.

\section{Availability of Data and Materials}

The datasets generated and/or analyzed during the current study are available from the corresponding author on reasonable request.

\section{References}

[1] Wijewardene, K., Mohideen, M.R., Mendis, S., et al. (2005) Prevalence of Hypertension, Diabetes and Obesity: Baseline Findings of a Population Based Survey in Four Provinces in Sri Lanka. Ceylon Medical Journal, 50, 62-70. https://doi.org/10.4038/cmj.v50i2.1571

[2] Katulanda, P., Ranasinghe, P., Jayawardena, R., et al. (2014) The Prevalence, Predictors and Associations of Hypertension in Sri Lanka: A Cross-Sectional Population Based National Survey. Clinical and Experimental Hypertension, 36, 484-491. https://doi.org/10.3109/10641963.2013.863321

[3] Kasturiratne, A., Pinidiyapathirage, M.J., Pathmeswaran, A., et al. (2011) Epidemiology of Hypertension in an Urban Population of Sri Lanka. Ceylon Medical Jour nal, 65, 256.

[4] Qaseem, A., Wilt, T.J., Rich, R., et al. (2017) Pharmacologic Treatment of Hypertension in Adults Aged 60 Years or Older to Higher versus Lower Blood Pressure Targets: A Clinical Practice Guideline From the American College of Physicians and the American Academy of Family Physicians. Annals of Internal Medicine, 166, 
430-437. https://doi.org/10.7326/M16-1785

[5] Bliziotis, I.A., Destounis, A. and Stergiou, G.S. (2012) Home versus Ambulatory and Office Blood Pressure in Predicting Target Organ Damage in Hypertension: A Systematic Review and Meta-Analysis. Journal of Hypertension, 30, 1289-1299. https://doi.org/10.1097/HJH.0b013e3283531eaf

[6] Hodgkinson, J., Mant, J., Martin, U., et al. (2011) Relative Effectiveness of Clinic and Home Blood Pressure Monitoring Compared with Ambulatory Blood Pressure Monitoring in Diagnosis of Hypertension: Systematic Review. British Medical Journal, 342, d3621. https://doi.org/10.1136/bmj.d3621

[7] Rustagi, N. and Singh, R. (2010) Mercury and Health Care. Indian Journal of Occupational and Environmental Medicine, 14, 45-48. https://doi.org/10.4103/0019-5278.72240

[8] Shah, A.S. (2012) Comparison of Mercury and Aneroid Blood Pressure Measurement in Youth. Paediatrics, 29, 1205-1210. https://doi.org/10.1542/peds.2011-3087

[9] Canzanello, V.J., Jensen, P.L. and Schwartz, G.L. (2001) Are Aneroid Sphygmomanometers Accurate in Hospital and Clinic Setting. Archives of Internal Medicine, 161, 729-731. https://doi.org/10.1001/archinte.161.5.729

[10] Pickering, T.G., Hall, J.E., Appel, L., et al. (2005) Recommendations for Blood Pressure Measurement in Humans and Experimental Animals. Hypertension, 45, 142-161. https://doi.org/10.1161/01.HYP.0000150859.47929.8e

[11] Sahu, D. and Bhaskaran, M. (2010) Palpatory Method of Measuring Diastolic Blood Pressure. Journal of Anaesthesiology Clinical Pharmacology, 26, 528-530.

[12] Pickering, T.G. (2003) What Will Replace the Mercury Sphygmomanometer? Blood Pressure Monitoring, 8, 23-25. https://doi.org/10.1097/00126097-200302000-00005

[13] Varughese, G.I. and Lip, G.Y.H. (2005) Good Bye Mercury? Blood Pressure Measurement and Its Future. Journal of the Royal Society of Medicine, 98, 89-90. https://doi.org/10.1177/014107680509800301

[14] Pinto, E. (2007) Blood Pressure and Ageing. Postgraduate Medical Journal, 83, 109114. https://doi.org/10.1136/pgmj.2006.048371

[15] Ravishi, C. and Messerli, G. (2016) Isolated Systolic Hypertension: An Update after SPRINT. American Journal of Medicine, 129, 1251-1258. https://doi.org/10.1016/j.amjmed.2016.08.032

[16] Bavishi, C., Bangalore, S., Messerli, F.H. (2017) Outcomes of Intensive Blood Pressure Lowering in Older Hypertensive Patients. Journal of the American College of Cardiology, 69, 486-493. https://doi.org/10.1016/j.jacc.2016.10.077

[17] Shahbabu, B., Dasgupta, A., Sarkar, K. and Sahoo, S.K. (2016) Which Is More Accurate in Measuring the Blood Pressure? A Digital or an Aneroid Sphygmomanometer. Journal of Clinical and Diagnostic Research, 10, 11-14.

[18] Orme, S., Ralph, S.G., Birchall, A., et al. (1999) The Normal Range for Inter-Arm Differences in Blood Pressure. Age Ageing, 28, 537-542. https://doi.org/10.1093/ageing/28.6.537

[19] Sharma, B. and Ramawat, P. (2016) Prevalence of Inter-Arm Blood Pressure Difference among Clinical Out-Patients. Journal of Environmental Research and Public Health, 10, 229-237. 


\section{List of Abbreviations:}

MSR Mercury Systolic blood pressure of Right arm

OSR Oscillometric Systolic blood pressure of Right arm

MDR Mercury Diastolic blood pressure of Right arm

ODR Oscillometric Diastolic blood pressure of Right arm

MSL Mercury Systolic blood pressure of Left arm

OSL Oscillometric Systolic blood pressure of Left arm

MDL Mercury Diastolic blood pressure of Left arm

ODL Oscillometric Diastolic blood pressure of Left arm

PR Pulse Rate 